broadening treatment options. CNS Spectrums, 7,636.

MAYFIELD, D., MCLEOD, G. \& HALL, P. (1974) The CAGE questionnaire: validation of a new alcoholism screening instrument. American Journal of Psychiatry, 131, 1121-1123.

McFARLANE, A. C. (1988) The longitudinal course of post-traumatic morbidity: the range of outcomes and their predictors. Journal of Nervous and Mental Disease, 176, 30-39.

NORTH, C. S., TIVIS, L., MCMILLEN, J. C. et al (2002a) Coping, functioning, and adjustment of rescue workers after the Oklahoma City bombing. Journal of Traumatic Stress, 15, 171-175.

NORTH, C. S., TIVIS, L., MCMILLEN, J. C et al (2002b) Psychiatric disorders in rescue workers after the Oklahoma City bombing. American Journal of Psychiatry, 159, 857-859.

SCHLENGER,W. E., CADDELL, J. M., EBERT, L., et al (2002) Psychological reactions to terrorist attacks: finding from the national study of American's reactions to September 11. JAMA, $\mathbf{2 8 8}$ $581-588$

SILVER, R. C., HOLMAN, E. A. McINTOSH, D. N., et al (2002) Nationwide longitudinal study of psychological responses to September 11. JAMA, $\mathbf{2 8 8}$

1235-1244

SPITZER, R. L., KROENKE, K. \& WILLIAMS, J. B.W. (1999) Validation and utility of a self-report version of PRIME$M D$ : the $P H Q$ primary care study. JAMA, 282, 1737-1744.
STREIMER, J. H., COSSTICK, J. \&

disaster site. Journal of Occupational (1985) The psychosocial adjustment of Australian Vietnam veterans. American Journal of Psychiatry, 142, 616-618.

TROUT, D., NIMGADE, A., MUELLER, C. et al (2002) Health effects and occupational exposures among office workers near the World Trade Center and Environmental Medicine 44, $601-605$

WAGNER, D., HEINRICHS, M. \& EHLERT U. (1998) Prevalence of symptoms of post-traumatic stress disorder in German professional firefighters. American Journal of Psychiatry, 155 1727-1732.

Craig L. Katz Clinical Assistant Professor, Department of Psychiatry, Mount Sinai School of Medicine, USA, Stephen Levin Associate Professor, Department of Community Medicine, Mount Sinai School of Medicine, USA, Robin Herbert Associate Professor, Department of Community Medicine, Mount Sinai School of Medicine, USA， *Simon Munro Specialty Registrar Year 4 Child and Adolescent Psychiatry, Child and Adolescent Mental Health Service, Ladyfield Offices, Glencaple Road, Dumfries, Scotland DG1 4TE, email: simon.munro@nhs.net, Anand Pandya Clinical Assistant Professor of

Psychiatry, New York University School of Medicine and President of the American National Alliance on Mental Illness, USA， Rebecca Smith Clinical Assistant Professor, Department of Psychiatry, Mount Sinai School of Medicine, NewYork, USA

\title{
Dementia screening in acute medical and geriatric hospital admissions
}

\author{
AIMS AND METHOD \\ We studied a representative cohort \\ of 161 patients over 65 years of age, \\ admitted non-electively to medical \\ and geriatric wards of a large \\ teaching hospital. Assessment for \\ dementia was made using DSM-IV \\ criteria. Psychiatric records were then \\ examined, masked, to determine the \\ involvement of psychogeriatric \\ services.
}

\author{
RESULTS \\ There were 111 possible cases of \\ dementia (69\%), of which $30(27 \%)$ \\ had prior local psychogeriatric case \\ notes; in 22 cases $(20 \%)$ the patient \\ had a prior psychiatric diagnosis of \\ dementia. Of 161 patients, 19 (12\%) \\ were seen by psychogeriatric services \\ during their admission, of whom 12 \\ (7\%) were already known to psychia- \\ tric services. Dementia was diag- \\ nosed in 17 (complicated by
}

\begin{abstract}
delirium in 2), depression in 1 and hypomania in 1. Many patients with a possible diagnosis of dementia had no psychiatric assessment.

CLINICAL IMPLICATIONS

Psychogeriatric assessment was performed on a minority of older people admitted to medical care. This population may include older people with undiagnosed dementia and unmet psychiatric care needs.
\end{abstract}

Dementia is a relatively common diagnosis among the elderly population. The frequency is thought to be higher in hospitalised patients than in community dwellers (Royal College of Psychiatrists, 2005), and is often unrecognised in the former group (Jorm \& Jolley, 1998; Laurila et al, 2004). Providing hospital care for the older person with dementia has implications for individual patient outcomes and broader financial costs.

Psychiatric assessment of older people in hospital may be an individual's first contact with mental health services, or may occur because specific review is required of a known patient. The aim of this study was to determine the proportion of elderly patients admitted to medical or geriatric wards thought to have a diagnosis of dementia, and their contact with local psychiatric services.

\section{Method}

An observational study of patients aged 65 years and over admitted to an acute medical unit or geriatric assessment unit generated 161 sets of patient details (80 acute medical admissions and 81 geriatric medical admissions). The patients were examined by the Department of Medicine for the Elderly (DoME) to determine potential diagnoses of dementia (using the DSM-IV checklist; see Appendix) (American Psychiatric Association, 1994) and delirium (using the Confusion Assessment Method; H. Hu, personal communication, 2006).

The demographic details of the 161 patients were then used by an assessor masked to patient diagnosis to enable a search of a computerised database, the Patient Information Management System, to determine those known to the local psychiatric services. Overall, 45 such 
patients were identified and the psychiatric case notes of 44 were examined by hand (one set of notes was missing). A further six patients had psychiatric case notes that were unavailable because they were outwith the catchment area.

Psychiatric diagnosis was sought along with relevant dates and follow-up where documented. Where data appeared incomplete, community psychiatric nurses were asked to confirm diagnosis using nursing notes. Once data had been collected from the psychiatric notes, psychiatric diagnoses given by the DoME were revealed. The diagnoses documented in the psychiatric case notes were compared with the diagnoses given by DoME staff. Dementia was the main diagnosis of interest, but other diagnoses were recorded.

\section{Results}

Of the 161 patients, 44 had available psychiatry case notes (Fig. 1). Of these 44 patients, 13 were men (30\%) and 31 were women (70\%). The mean age of the cohort was 80 years and the mean age of patients known to psychiatric services was also 80 years.

From the medical cohort of 161 patients, 111 had a likely diagnosis of dementia as defined by the DSM-IV checklist (69\%). Of these 111 , only 22 (20\%) had locally available psychiatric case notes documenting a diagnosis of dementia. Of the 44 patients with psychiatric case notes, 20 were known to services prior to the index hospital admission and 19 were referred as new cases. Psychiatric diagnoses are given in Table 1.

Medical staff referred five patients for psychiatric review who were never seen by psychiatry and whose psychiatric case notes contained no information regarding follow-up. Proposed diagnoses by medical staff for these individuals were dementia in three cases. There was disagreement between the DoME proposed diagnosis of dementia and the diagnosis given by psychiatric staff in two cases: the psychiatric staff diagnosed depression in

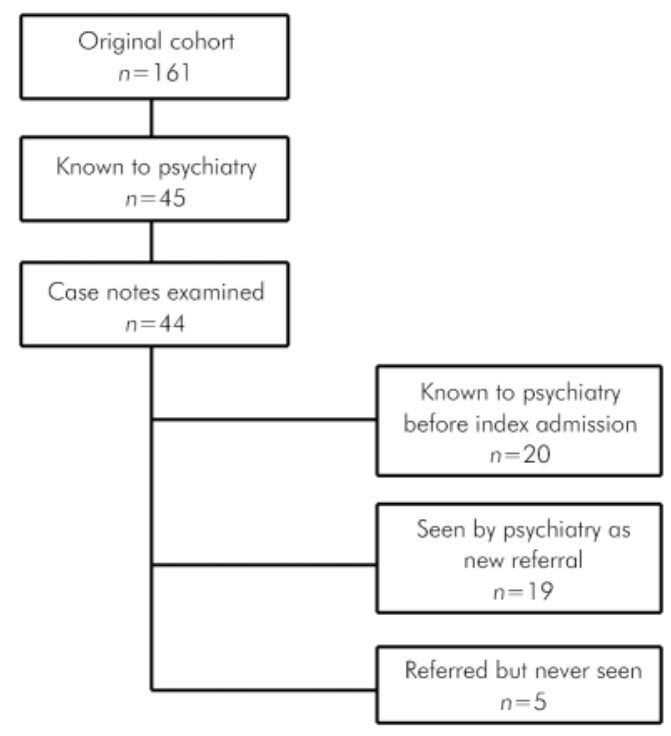

Fig. 1. Study profile.
Table 1. Psychiatric diagnoses in the sample

\begin{tabular}{lcc}
\hline & $\begin{array}{c}\text { Patients already } \\
\text { known to } \\
\text { psychiatric } \\
\text { services }(n=20)\end{array}$ & $\begin{array}{c}\text { Patients } \\
\text { referred as new } \\
\text { cases }(n=19)\end{array}$ \\
Diagnosis & $n$ & $n$ \\
\hline Dementia & 12 & 15 \\
Depression & 6 & 1 \\
Dementia with delirium & 0 & 2 \\
Psychotic depression & 1 & 0 \\
Hypomania secondary to & & 1 \\
steroid use & 1 & \\
\hline
\end{tabular}

original papers

one case and hypomania secondary to steroid use in the other.

\section{Discussion}

In this sample, $20 \%$ of patients considered by DoME staff to have dementia were known to psychiatric services as having dementia. A minority were referred during the study period as new cases. It would seem that the majority of individuals with dementia in our sample were being managed by primary care and other services without ever coming to the attention of mental health services. It is possible that some patients were not known to health or social services at all and that admission to a medical ward was the first time a diagnosis of dementia had been considered. There is potentially substantial unmet clinical need. It could be argued that involvement with psychiatric services is not always necessary when a person has dementia but only when particular problems arise. It seems likely that the diagnosis of dementia is not solely determined by psychiatrists and that sharing of this information between professionals is not universal.

The DoME and psychiatric staff might have used different screening tools with potentially different validity to reach diagnosis. The DSM-IV checklist was used by DoME staff to define cases, but psychiatric notes generally did not contain information as to how the diagnosis had been reached. It is likely that diagnoses by psychiatric staff were based on the Mini Mental State Examination (Folstein et al, 1975) and clinical presentation. There may be subtle differences in the way in which diagnosis was reached, as the psychiatric assessment could have been made by a liaison nurse, a consultant psychiatrist or a specialist registrar; it was not obvious from perusing the notes which professional had carried out the assessment.

There was disagreement about diagnosis in some cases. Dementia was diagnosed by DoME staff in one case in which psychiatric staff diagnosed depression and in one case thought to be an organic state secondary to steroid use. It would be difficult to be certain about these individuals' diagnoses without accurate longitudinal information which is not yet available. Five patients were referred for psychiatric assessment but were never seen by psychiatric services; three of these patients were thought to have dementia. There are implications for 
7

original papers patients who 'slip through the net'. It was not possible from examining the notes to determine whether these individuals were later picked up by primary care or social work services.

The proportion of patients in medical and geriatric wards thought to have dementia in the original sample was high, with a point prevalence of $69 \%$. This is greater than the prevalence of $5-45 \%$ (mean prevalence $31 \%$ ) mentioned in the Who Cares Wins document from the Royal College of Psychiatrists (2005). This study population may, therefore, not be entirely typical of older people in hospital. It is also possible that there is overdiagnosis of dementia in this sample due to observational bias or reliance on the DSM-IV checklist.

Hospital care or DoME admission could represent a default pathway whereby older people with dementia come into hospital when a crisis arises at home. It is possible that the DoME is more accessible than psychogeriatric services, especially out of hours. Relatives and carers may be more familiar with the idea of being sent to the accident and emergency department with their loved one, rather than relying on the referrer contacting a community psychiatric nurse or psychiatrist for telephone advice.

This study may have limitations because of the small number of psychiatric case notes examined. The six outof-area case notes were not examined for pragmatic reasons and it is not possible to comment how the final results might have been affected.

Future research could examine if these results are similar to findings in other geographical locations and whether the model of psychiatric liaison provided has any impact on numbers of patients seen. Longitudinal information on service use and individual outcomes would be interesting to determine if there is a key time when psychiatric input becomes most valuable. Follow-up information on the cases where there is disagreement on diagnosis may be of educational value.

The optimal balance between confidentiality and coordinated team working may be difficult to determine, but could offer benefits to individual patients by avoiding hospital admission, shortening length of stay and improving outcomes. Providing expert advice on diagnosis and management has scope to improve care for some of our most vulnerable elderly people.

\section{Appendix}

\section{DSM-IV dementia checklist}

For a diagnosis of dementia to be made according to DSM-IV all criteria A to D must be satisfied.
A. The presence of multiple cognitive deficits, one of which must include memory impairment.

B. At least one of the following cognitive disturbances:

- dysphasia

- dyspraxia

- agnosia

- disturbance of executive functioning.

C. The above symptoms must be sufficiently severe to cause significant impairment in the patient's social or occupational functioning and represent a decline from previous level of functioning.

D. Delirium must be excluded.

\section{Declaration of interest}

None.

\section{Acknowledgements}

Many thanks to the staff of the Glasgow Royal Infirmary academic department for providing the original data. We thank Professor Stott for his encouragement and advice on editing. Thanks are also due to the secretarial staff at Parkview Resource Centre and the records staff at Parkhead Hospital for sourcing case notes needed for the study.

\section{References}

AMERICAN PSYCHIATRIC

ASSOCIATION (1994) Diagnostic and

Statistical Manual of Mental Disorders (4th edn) (DSM-IV). APA .

FOLSTEIN, M. F., FOLSTEIN, S. E. \& McHUGH, P. R. (1975) Mini Mental

State: a practical method for grading the cognitive state of patients for the clinician. Journal of Psychiatric

Research, 12, 189-198.

JORM, A. F. \& JOLLEY, D. (1998)

The incidence of dementia: a metanalysis. Neurology, $\mathbf{5 1}$ $728-733$

LAURILA, J.V., PITKALA, K. H., STRANDBERG, T. E., et al (2004) Detection and documentation of dementia and delirium in acute geriatric wards. General Hospital Psychiatry, 26, 31-35.

ROYAL COLLEGE OF PSYCHIATRISTS of Psychiatrists. (2005) Who Cares Wins. Royal College

*Alison Gordon Specialist Registrar in Psychiatry of Old Age, 4th Floor Atholl House, Churchill Avenue, East Kilbride, Lanarkshire G74 1LU, email: Alison. Gordon@lanarkshire.scot.nhs.uk, Hilda Hu Specialist Registrar in Old Age Medicine, Glasgow Royal InfirmaryAcademic Department, Anthony Byrne Specialist Registrar in Old Age Medicine, Glasgow Royal Infirmary, David J. Stott Professor of Old Age Medicine, Glasgow Royal Infirmary, Glasgow 